University of Nebraska - Lincoln

DigitalCommons@University of Nebraska - Lincoln

March 1977

\title{
Electron tunneling, charge transfer, and the intermolecular forces between two $\mathrm{H}_{2}$ molecules
}

Gordon A. Gallup

UNL,ggallup1@unl.edu

Follow this and additional works at: https://digitalcommons.unl.edu/physicsgallup

Part of the Physics Commons

Gallup, Gordon A., "Electron tunneling, charge transfer, and the intermolecular forces between two $\mathrm{H}_{2}$ molecules" (1977). Gordon Gallup Publications. 16.

https://digitalcommons.unl.edu/physicsgallup/16

This Article is brought to you for free and open access by the Research Papers in Physics and Astronomy at DigitalCommons@University of Nebraska - Lincoln. It has been accepted for inclusion in Gordon Gallup Publications by an authorized administrator of DigitalCommons@University of Nebraska - Lincoln. 


\title{
Electron tunneling, charge transfer, and the intermolecular forces between two $\mathrm{H}_{2}$ molecules
}

\author{
G. A. Gallup \\ Department of Chemistry, University of Nebraska, Lincoln, Nebraska 68588 \\ (Received 19 January 1976) \\ The spherically averaged van der Waals potential for the system $\mathrm{H}_{2}-\mathrm{H}_{2}$ has been calculated using a \\ nonorthogonal CI method based on SCF orbitals for the individual molecules. With suitable restrictions on \\ the CI calculation we have isolated various contributions to the van der Waals potential, and have found \\ that charge transfer and dispersion effects are comparable to distances corresponding to the potential \\ minimum. The charge transfer contribution is further discussed in terms of a mechanism involving electron \\ tunneling through the intervening space between the molecules. Our calculation gives a spherically averaged \\ van der Waals well depth of $2.96 \mathrm{meV}$ at $3.49 \AA$. Experimental values are $3.00 \mathrm{meV}$ at $3.49 \AA$
}

\section{INTRODUCTION}

Attempts to elucidate the nature of the forces between molecules have occupied many workers over a long period of time. The treatises by Hirschfelder, Curtiss, and Bird ${ }^{1}$ and by Margenau and Kestner ${ }^{2}$ give complete discussions of most aspects of the problems. The latter work gives an excellent historical perspective.

Quantum mechanical investigations commenced with the work of Epstein ${ }^{3}$ and Eisenschitz and London. ${ }^{4}$ In the ensuing years both the perturbation and the variational approaches were used to examine intermolecular forces. As the simplest molecule-molecule system, the $\mathrm{H}_{2}-\mathrm{H}_{2}$ pair has engaged the attention of many workers. Perturbation techniques have been used since early times, ${ }^{5-9}$ while the development of computers over the last 15 years has recently increased the use of the variational approach. ${ }^{10-15}$ The AO bases used in these calculations have varied from a minimal set consisting of a $1 s$ orbital at each proton to the $2 s 1 p$ set of Bender and Schaefer. ${ }^{12}$ The last study is of particular interest to us, and we wish to examine the results closely.

Bender and Schaefer report on both a full and a restricted $\mathrm{CI}$ calculation for $\mathrm{H}_{2}-\mathrm{H}_{2}$ in linear configurations. Both calculations show an apparent van der Waals minimum of depth $1.89 \mathrm{meV}$ at $3.78 \AA$ distance between the centers of mass. The experimental values ${ }^{16}$ are $R=3.49 \AA$ and $\epsilon=3.00 \mathrm{meV}$, for the spherically averaged potential. Although the calculation and experiment refer to very different situations, Bender and Schaefer attribute the discrepancy to "the known incompleteness of ... [the] basis." Their explanation ignores the electrostatic interaction of two $\mathrm{H}_{2}$ molecules, however.

$\mathrm{H}_{2}$ is an axial quadrupole ${ }^{17}$ with a moment $Q=0.38$ a. $u{ }^{18}$ When two axial quadrupoles are colinear, the mutual potential is at its repulsive maximum and one has

$$
V=6 Q^{2} / R^{5} \quad \text { (linear configuration) . }
$$

If the above linear geometry calculation is corrected for the quadrupole-quadrupole repulsion, a closer agreement with the spherically averaged experimental results is expected. This indeed happens, and a spherically averaged well depth of $-2.62 \mathrm{meV}$ at $3.44 \AA$ is estimated from the calculations. The depth is still too small, and, in addition, excludes any effects of the change in quadrupole moment when the two molecules are mutually polarized by one another. Nevertheless, the gloomy assessment of the basis appears overdone.

In this article we examine the theoretical spherically averaged van der Waals potential for $\mathrm{H}_{2}-\mathrm{H}_{2}$ by calculating the $R$ dependence of the energy for four different orientations of the two molecules, and obtaining the spherical average numerically. We use a method involving a nonorthogonal CI constructed with basis functions which are SCF orbitals for an individual $\mathrm{H}_{2}$, and therefore, completely localized to one of the molecules. This basis is of the kind typically used in perturbation treatments of long range forces, and its use allows us to analyze separately the physical nature of the many contributions to van der Waals forces. Wormer and van der Avoird ${ }^{19}$ have recently done calculations very similar to this on the ethylene dimer.

\section{QUANTUM MECHANICAL CALCULATIONS}

In recent calculations on $\mathrm{H}_{3}$ Norbeck and Certain ${ }^{20}$ carefully optimized the scale factors for a $1 s, 1 s^{\prime}, 2 p$ set for $\mathrm{H}_{2}$. We have adapted this basis to our use, with the single modification of replacing the $1 \mathrm{~s}$ ten contracted Gaussian functions used in Ref. 20 with a five Gaussian function period due to Whitten. ${ }^{21}$ Table I shows the SCF and MOCI energies of this basis compared to values from other studies and that the five Gaussian $1 \mathrm{~s}$ function is only slightly inferior to the $1 s$ function used in Ref. 20.

Figure 1 shows the four geometric orientations used in this study. Calculations were made at intermolecular distances of $6.0,6.6,7.2,7.8,10.0$, and 50.0 a.u. for each arrangement.

The AO's used here give rise to 10 MO's for each $\mathrm{H}_{2}$ molecule, $1 \sigma_{g}, 2 \sigma_{g}, 3 \sigma_{g}, 1 \sigma_{u}, 2 \sigma_{u}, 3 \sigma_{u}, 1 \pi_{u x}, 1 \pi_{u y}, 1 \pi_{g x}$, and $1_{\pi_{g y}}$. We use a subscript 1 or 2 to designate the location of the orbital on molecule 1 or molecule 2 . The principal configuration for the system is $\left(1 \sigma_{z 1}\right)^{2}\left(1 \sigma_{z 2}\right)^{2}$, which at infinite separation has twice the SCF energy of Table I.

For our CI calculation we have used the 12 SCF orbitals $1 \sigma_{s 1}, 1 \sigma_{z 2}, 1 \sigma_{u 1}, 1 \sigma_{u 2} 2 \sigma_{u 1}, 2 \sigma_{u 2}, 11_{u x 1}, 1 \pi_{u x 2}, 1_{\pi_{u y 1}}$, and $1 \pi_{u y 2}$, augmented with six Rydberg-like orbitals $r \sigma_{u 1}$, 
TABLE I. Comparison of various calculations of the energy of the $\mathrm{H}_{2}$ molecule.

\begin{tabular}{llcl}
\hline \hline Basis & Reference & SCF & Full CI \\
\hline $1 s, 1 s^{\prime}$ & 12 & $\cdots$ & -1.151 \\
$1 s, 1 s^{\prime}, 2 p$ & 14 & -1.13119 & -1.16570 \\
$1 s, 1 s^{\prime}, 2 p$ & This work & -1.13281 & -1.16945 \\
$1 s, 1 s^{\prime}, 2 p$ & 20 & -1.13324 & -1.17001 \\
Exact & 18 & -1.13364 & -1.17447 \\
\hline \hline
\end{tabular}

$r \sigma_{u 2}, r_{\pi_{u x 1}}, r_{\pi_{u x 2}}, r_{\pi_{u y 1}}$, and $r_{\pi_{u y 2}}$. The latter orbitals are single component $p$ type Gaussians located at the center of the mass of each $\mathrm{H}_{2}$ and have a scale factor of 0.125 . This value gives rough correspondence to hydrogenic $2 p$ orbitals and was not further optimized. The maximum of such orbitals occurs around 2 a.u. from the center, somewhat outside the normal boundaries of the $\mathrm{H}_{2}$ molecule. The CI calculation consists of all single and double excitations out of the principal configuration into the remaining 16 orbitals. Values of the energy for each of the geometrical arrangements are given in Table II.

We assume that the angular dependence of $V$ may be expressed as

$$
\begin{aligned}
V\left(R, \theta_{1}, \theta_{2}, \phi\right)= & V_{\mathrm{sph}}(R)+A\left[P_{2}^{(0)}\left(\cos \theta_{1}\right)+P_{2}^{(0)}\left(\cos \theta_{2}\right)\right] \\
& +B P_{2}^{(0)}\left(\cos \theta_{1}\right) P_{2}^{(0)}\left(\cos \theta_{2}\right) \\
& +C P_{2}^{(1)}\left(\cos \theta_{1}\right) P_{2}^{(1)}\left(\cos \theta_{2}\right) \cos \phi \\
& +D P_{2}^{(2)}\left(\cos \theta_{1}\right) P_{2}^{(2)}\left(\cos \theta_{2}\right) \cos 2 \phi,
\end{aligned}
$$

where $P_{l}^{(m)}$ is a conventionally defined associated Legendre function, $\phi$ is the dihedral angle between the two molecules, and $\theta_{i}$ is the angle between molecule $i$ and the line connecting the two centers of mass. A more complete discussion of the angular dependence of the $\mathrm{H}_{2}-\mathrm{H}_{2}$ potential appears elsewhere. ${ }^{19}$

It is not difficult to show that the four geometries calculated are sufficient to determine $V_{\text {sph }}$ for a function of the form of (2). Referring to Fig. 1 for the meaning of the subscripts we have

$$
V_{\mathrm{sph}}(R)=\left[-E_{\mathrm{I}}(R)+4 E_{\mathrm{II}}(R)+4 E_{\mathrm{III}}(R)+2 E_{\mathrm{IV}}(R)\right] / 9,
$$

and values of this spherical average are also given in Table II. The depth of the van der Waals well here is $2.96 \mathrm{meV}$ which compares very favorably with the recent molecular scattering results of $3.00 \mathrm{meV}{ }^{16}$

In addition to the CI resulting from all single and double excitations out of $\left(1 \sigma_{\ell 1}\right)^{2}\left(1 \sigma_{g 2}\right)^{2}$, we performed cal-
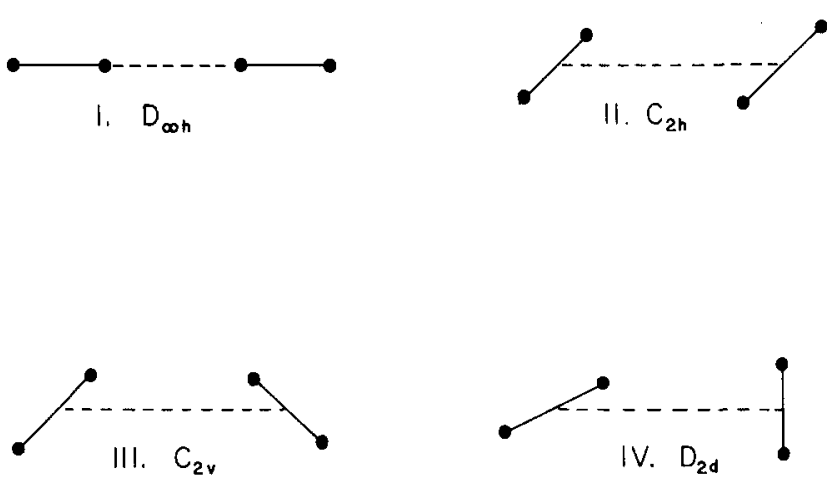

FIG. 1. Geometrical arrangement of the two $\mathrm{H}_{2}$ molecules for the calculation of the spherical average.

culations with restrictions. This was done to test for the importance of various contributions to the van der Waals potential by an analysis similar to perturbation theory treatments.

A basis such as ours, with each orbital strictly localized to one or the other of the molecules, possesses a natural classification of each of the excited configurations into different types involving different physical phenomena and with varying contributions to the intermolecular potential.

Examination of the configurations shows evidence of two main types: neutral configurations $(N)$, in which there are two electrons associated with each of the molecules, and charge transfer configurations $(C)$, in which there are net charges at each of the molecules. We consider the $N$ class first.

The category of neutral configurations may be further subdivided into those producing dispersion forces, $N_{D}$, and those giving the distortion of the molecules and the electron correlation within the molecules, $N_{t}$. In practice the $N_{D}$ class involves simultaneous single excitations at each molecule, and the $N_{I}$ class single and double excitations at just one molecule. Physically, the classification places the ground configuration with the $N_{I}$ group.

The $C$ category also has two subclasses, but here we use the net charge as the criterion. $C_{1}$ contains those configurations with \pm 1 net electrical charge at the molecules and $C_{2}$, those with \pm 2 net electrical charges. The $C$ category could perhaps be subdivided on a different basis, but various physical criteria which might be used do not apply well to $\mathrm{H}_{2}$ molecules themselves, but rather to $\mathrm{H}_{2}^{ \pm}$and more highly charged species. In $\mathrm{Ta}-$

TABLE II. Energies for four mutual orientations of two $\mathrm{H}_{2}$ molecules and the spherical average. Energies and distances given in atomic units.

\begin{tabular}{rlcccc}
\hline \hline$R$ & $E_{\mathrm{I}}$ & $E_{\mathrm{II}}$ & $E_{\mathrm{III}}$ & $E_{\mathrm{IV}}$ & $E_{\text {8ph }}$ \\
\hline 6.0 & -2.3310626 & -2.3313605 & -2.3312154 & -2.3313585 & -2.3313287 \\
6.6 & -2.3312869 & -2.3314379 & -2.3313506 & -2.3314167 & -2.3314112 \\
7.2 & -2.3313349 & -2.3314223 & -2.3313656 & -2.3313999 & -2.3314018 \\
7.8 & -2.3313360 & -2.3313898 & -2.3313517 & -2.3313701 & -2.3313744 \\
10.0 & -2.3313074 & -2.3313207 & -2.3313099 & -2.3313123 & -2.3313155 \\
50.0 & -2.3313025 & -2.3313023 & -2.3313029 & -2.3313022 & -2.3313025 \\
\hline \hline
\end{tabular}


TABLE III. Numbers of configurations corresponding to each of the classifications discussed in the text.

\begin{tabular}{llllll}
\hline \hline Orientation & $N_{I}$ & $N_{D}$ & $C_{1}$ & $C_{2}$ & Total \\
\hline I & 18 & 26 & 44 & 13 & 101 \\
II & 31 & 46 & 88 & 24 & 189 \\
III & 30 & 49 & 86 & 24 & 189 \\
IV & 21 & 22 & 46 & 15 & 104 \\
\hline
\end{tabular}

ble III we give the number of symmetry functions in each of the classifications.

The spherical average energy for the calculations with the configuration sets $N_{I}, N_{I}+N_{D}, N_{I}+C_{1}, N_{I}+C_{2}$, have been determined, and Table IV gives the energies of $N_{I}$ and the contributions due to $N_{D}, C_{1}$, and $C_{2}$, obtained by difference assuming additivity. The sums of these four energies give the correct value within $10 \%$. The error corresponds to interactions between the groups from third order and higher perturbation terms. Figure 2 shows curves for these contributions, and the correct total.

\section{DISCUSSION}

Previous numerical perturbation treatments of the van der Waals forces in $\mathrm{H}_{2}-\mathrm{H}_{2}$ have neglected charge transfer phenomena completely. It is, of course, implicit in MOCI calculations, but mixed inextricably with other phenomena. Murrell, Randic, and Williams ${ }^{23}$ in their general theory of intermolecular forces include such terms, but seem to feel that the contributions are small. Certain and Hirschfelder ${ }^{24}$ have also discussed such charge effects in terms of perturbation theory, with applications to $\mathrm{H}_{2}^{+}$and $\mathrm{H}_{2}$.

Examination of the present results show that charge transfer, particularly those of set $C_{1}$, and dispersion effects have nearly equal contributions for our basis.

From Table IV it is seen that the contribution from the $C_{2}$ configurations is minimal throughout the range of interest here. We say no more about these.

There are a large number of $C_{1}$ configurations, and it is of considerable interest to see which ones predominate. However, a quick examination of the results indicate that no one of these configurations stands out, and the relatively large contribution of $C_{1}$ is an accumulative effect due to all. There does seem to be some preference of single over double excitation configurations, but the difference is small.

The contribution from $N_{D}$ configurations is just the well understood London dispersion energy. This our basis; there is only partial contribution from dipolequadrupole and quadrupole-quadrupole dispersion terms. To get all of this would require addition to the basis of orbitals with local $\pi_{g}$ and $\delta_{g}$ symmetry. A test calculation at $R=6.6$ a. u. was made using $\pi_{\varepsilon}$ orbitals and the energy was lowered only $0.1 \mathrm{meV}$, and such orbitals were not used further.

The value of the dispersion energy is closely equal to the total energy at the minimum. It would appear

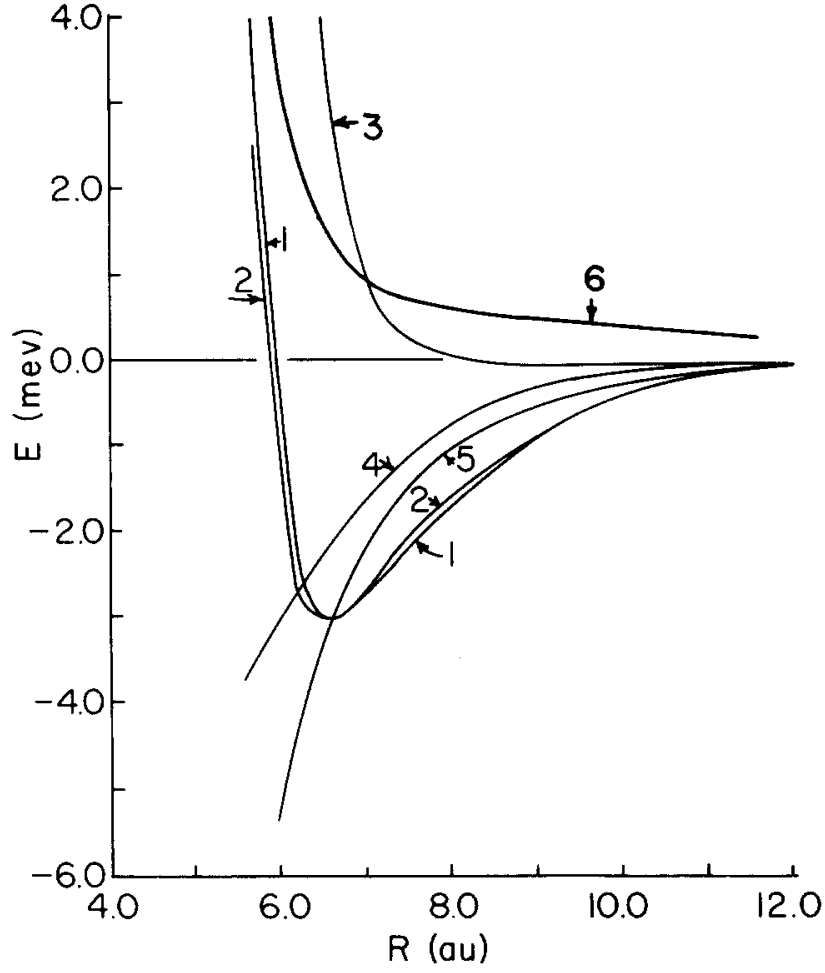

FIG. 2. Vaxious contributions and the total energy of the spherically averaged mutual potential of two $\mathrm{H}_{2}$ molecules. (1) Calculated, this work; (2) experimental, Ref. 14; (3) repulsive potential, configuration class $N_{I}$; (4) charge transfer, configuration classes $C_{1}+C_{2}$; (5) dispersion contribution, configuration class $N_{D}$; (6) spherically averaged SCF energy.

that this fact explains in part the success that previous workers have had in calculating the depth of the van der Waals well without including charge transfer effects. It is also seen that the $N_{I}$ configurations (which include the first order effects) show a strong, long distance repulsion. This is due in part to the inclusion of the diffuse orbitals in the basis. However, the diffuse orbitals also contribute to dispersion energy, and so there is a tendency for the effects to cancel. The same cancellation occurs to a somewhat lesser extent with the charge transfer.

It is reasonable to conclude that previous calculations of the van der Waals forces in $\mathrm{H}_{2}-\mathrm{H}_{2}$ have been successful because of cancellation of errors. We also cannot claim that our calculation is completely free from this problem, but the calculation of both the attractive and repulsive parts of the potential have been pushed closer to their correct values.

TABLE IV. Spherically averaged contributions to the energy from each configuration type. All energies and distances given in atomic units.

\begin{tabular}{rcccc}
\hline \multicolumn{1}{l}{$R$} & $N_{I}$ & $N_{D}$ & $C_{1}$ & $C_{2}$ \\
\hline 6.0 & -2.3310252 & -0.0002101 & -0.0001162 & -0.0000042 \\
6.6 & -2.3312219 & -0.0001213 & -0.0000852 & -0.0000020 \\
7.2 & -2.3312795 & -0.0000681 & -0.0000553 & -0.0000009 \\
7.8 & -2.3312972 & -0.0000423 & -0.0000364 & -0.0000004 \\
10.0 & -2.3313034 & -0.0000094 & -0.0000028 & 0.0 \\
50.0 & -2.3313025 & 0.0 & 0.0 & 0.0 \\
\hline \hline
\end{tabular}




\section{ELECTRON TUNNELING AND CHARGE TRANSFER}

In his classic discussion of the theory of charge transfer complexes, Mulliken ${ }^{25}$ suggests that forces due to charge transfer may be competitive with dispersion forces even between like molecules. Our results appear to verify this early prediction.

However, the inclusion of charge transfer configurations in intermolecular force calculations has been criticized recently. The general problem, arising in any LCAO basis calculation which compares energies at two or more geometries, has been called the basis set enhancement errors. ${ }^{26}$ In the simplest molecular interaction cases, the error is considered to occur because the quality of the basis for one molecule in a composite system is affected by the presence of the basis located at the other molecule, and depends upon distances between molecules. It is convenient to consider the enhancement error at two levels.

The first level was first identified by Kestne ${ }^{27}$ in interpreting correctly the spurious minimum in the $\mathrm{He}_{2}$ SCF calculation of Ransil. ${ }^{28}$ A similar spurious well can be produced in a minimal $1 s$ basis calculation of the interatomic potential of the ${ }^{3} \sum_{u}^{+}$state of $\mathrm{H}_{2}$ by the simple expedient of using (for example) $\zeta=0.9$ at all distances. This value of $\zeta$ is not optimum for infinite separations but is appropriate for a smaller distance. We emphasize that the minimum produced in such a calculation is completely unconnected with the real van der Waals well in ${ }^{3} \mathrm{~S}_{u}^{+} \mathrm{H}_{2}$.

Wormer and van der Avoir $\mathrm{d}^{19}$ have suggested a test for the enhancement effect. They propose to calculate the energy of one molecule in the presence of the empty basis from the other. This test is easily applied to ${ }^{3} \sum_{u}^{+} \mathrm{H}_{2}$ and shows the expected effect: the calculated energy of a single $\mathrm{H}$ atom is improved by the presence of the extra $1 s$ orbital for $\zeta \neq 0.1$. This shows, by counterexample, that the test is not sufficient, however. The spurious minimum in $\mathrm{H}_{2}$ occurs only for $\zeta<1$, whereas the test is positive for either $\zeta<1$ or $\zeta>1$. The question of necessity of the test is unanswered.

It is fortunate that this level of difficulty with the enhancement error shows at the SCF level. Figure 2 also includes the spherical average SCF energy for our basis, and there is no evidence of a minimum. In our calculation the scale values of the orbitals are optimal for separated $\mathrm{H}_{2}$. Tests at several distances and geometries showed that the optimum values changed by less than $1 \%$ throughout the whole range of our calculations.

The second level of possible enhancement error is much more subtle, occurring only with CI calculations and affecting electron correlation. The error here has been called distance dependent intramolecular electron correlation, and happens if the orbitals of a second molecule provide additional flexibility for electron correlation over that provided by the basis for the first molecule. Some workers suggest the elimination of the offending configurations to overcome the problems. ${ }^{9,19}$ In our case, the configurations giving these effects are the $C_{1}$ and $C_{2}$ groups, and as before we ignore the $C_{2}$ set.

The analysis here is very complicated, since reasonable arguments lead to the conclusion that real physical processes occur, requiring $C_{1}$ configurations for their correct description. The well-known phenomenon of tunneling allows the electrons from one molecule to get into the region of the other. Classical or quantal descriptions suggest that the electron will spend some time with the other molecule before returning. Recent experiments indicate that slow electrons are captured momentarily by molecules. ${ }^{29}$ Similar captures are ex-. pected to occur between pairs of molecules separated by distances near the van der Waals minimum. These physical processes allow for a certain amount of electron delocalization, which also contributes to the attractive well between the two molecules. All of these phenomena are expected to react upon the electron correlation within the molecules, and hence cause a real distance dependent intramolecular correlation effect to occur.

$C_{1}$ configurations can thus contribute both to real effects and to a possible enhancement errors. An even crude estimate of the division here appears to present great difficulties. In their ethylene calculation, Wormer and van der Avoird ${ }^{19}$ agree that their enhancement error test is too severe and overestimates the problem at the second level. Further study is needed to settle the question.

As was mentioned above, some workers have chosen to eliminate these terms. We have included them in our calculation. It appears that no rationale for a compromise between these two positions is available today.

${ }^{1}$ J. O. Hirschfelder, C. F. Curtiss, and R. B. Bird, Molecular Theory of Gases and Liquids (Wiley, New York, 1954).

${ }^{2}$ H. Margenau and N. R. Kestner, Theory of Intermolecular Forces, 2nd ed. (Pergamon, New York, 1971).

${ }^{3}$ P. S. Epstein, Proc. Nat. Acad. 12, 629 (1926); 13, 432 (1927).

${ }^{4} R$. Eisenschitz and F. London, Z. Phys, 60, 491 (1930).

${ }^{5} \mathrm{~J}$. deBoer, Physica 9, 363 (1942).

${ }^{6}$ H. Margenau, Phys. Rev. 64, $131(1943)$; A. A. Evett and H. Margenau, ibid. 90, 1021 (1953).

${ }^{7}$ E. A. Mason and J. O. Hirschfelder, J. Chem. Phys. 26, 756 (1957).

${ }^{8}$ E. Kochanski, B. Roos, P. Seighahn, and M. H. Wood, Theor. Chim. Acta 32, 151 (1973); E. Kochanski and J. F. Gouyet, ibid. 39, 329 (1975).

${ }^{9}$ J. P. Daudey, J. P. Malrieu, and O. Rojas, Int. J. Quantum Chem. 8, 17 (1974),

${ }^{10} \mathrm{~V}$. Magnasco and G. F. Musso, J. Chem. Phys. 46, 4015 (1967); 47, 1723, 4629 (1967); 48, 2657 (1968).

${ }^{11}$ V. Magnasco, G. F. Musso, and R. McWeeny, J. Chem. Phys. 47, 4617 (1967).

${ }^{12}$ M. Rubinstein and I. Shavitt, J. Chem. Phys. 51, 2014 (1969).

${ }^{13}$ C. W. Wilson and W. A. Goddard, J. Chem. Phys. 51, 716 (1969); 56, 5913 (1972).

${ }^{14}$ C. F. Bender and H. F. Schaefer, J. Chem. Phys. 57, 217 (1972).

${ }^{15}$ R. W. Patch, J. Chem. Phys. 59, 6468 (1973).

${ }^{16}$ M. G. Dondi, U. Valbusa, and G. Scoles, Chem. Phys. Lett. 17, 137 (1972); J. M. Farrar and Y. T. Lee, J. Chem. 
Phys. 57, 5492 (1972).

${ }^{17}$ See for example, C. J. Böttcher, Theory of Electric Polarization (Elsevier, New York, 1973).

${ }^{18}$ W. Kolos and L. Wolniewicz, J. Chem. Phys. 43, 2429 (1965).

${ }^{19}$ P. E. S. Wormer and A. van der Avoird, J. Chem. Phys. 62, 3326 (1975).

${ }^{20}$ J. M. Norbeck and P. R. Certain, J. Chem. Phys, 63, 4127 (1975).

${ }^{21}$ J. L. Whitten, J. Chem. Phys. 39, 349 (1963).

${ }^{22}$ G. A. Gallup, Mol. Phys. (to be published).
${ }^{23} J$. N. Murrell, M. Randic, and D. R. Williams, Proc. R. Soc. (London) Ser. A 284, 566 (1964).

${ }^{24} \mathrm{P}$. R. Certain and J. O. Hirschfelder, J. Chem. Phys. 52, $5992(1970)$.

${ }^{25}$ R. S. Mulliken, J. Am. Chem. Soc. 74, 811 (1952); J. Chem. Phys. 19, 514 (1951).

${ }^{26}$ P. E. S. Wormer, T. van Berkel, and A. van der Avoird, Mol. Phys. 29, 1181 (1975).

${ }^{27}$ N. R. Kestner, J. Chem. Phys. 48, 252 (1968).

${ }^{28}$ B. J. Ransil, J. Chem. Phys. 34, 2109 (1961).

${ }^{29}$ P. D. Burrow, Chem. Phys. Lett. 36, 594 (1975). 